\title{
RELAXED ORDERED SUBSETS ALGORITHM FOR IMAGE RESTORATION OF CONFOCAL MICROSCOPY
}

\author{
Saowapak Sotthivirat and Jeffrey A. Fessler \\ Dept. of Electrical Engineering and Computer Science \\ University of Michigan, Ann Arbor, MI 48109-2122
}

\begin{abstract}
The expectation-maximization (EM) algorithm for maximumlikelihood image recovery converges very slowly. Thus, the ordered subsets EM (OS-EM) algorithm has been widely used in image reconstruction for tomography due to an orderof-magnitude acceleration over the EM algorithm. However, OS-EM is not guaranteed to converge. The recently proposed ordered subsets, separable paraboloidal surrogates (OS-SPS) algorithm with relaxation has been shown to converge to the optimal point while providing fast convergence. In this paper, we develop a relaxed OS-SPS algorithm for image restoration. Because data acquisition is different in image restoration than in tomography, we adapt a different strategy for choosing subsets in image restoration which uses pixel location rather than projection angles. Simulation results show that the order-of-magnitude acceleration of the relaxed OS-SPS algorithm can be achieved in restoration. Thus the speed and the guarantee of the convergence of the OS algorithm is advantageous for image restoration as well.
\end{abstract}

\section{INTRODUCTION}

Statistical techniques have been shown to improve image quality in image restoration. Since closed form solutions for these techniques are usually unobtainable, iterative algorithms are needed. However, there are some drawbacks of existing algorithms such as convergence, computation time, and parallelizability.

The expectation-maximization (EM) algorithms [1,2] and their ordered subset (OS) version [3] are among the most common used algorithms; however, they have some limitation either on speed or convergence. The EM algorithms are guaranteed to converge; however, they converge very slowly. The OS-EM algorithm [3] has become very attractive to image reconstruction in tomography due to its fast convergence rate compared with the EM algorithms. However, the OS-EM algorithm is not guaranteed to converge. Therefore, many approaches have been proposed to solve the convergence problem of the $O S$ algorithm such as the row-action maximum likelihood algorithm (RAMLA)
[4] and its regularized version, the block sequential regularized EM (BSREM) algorithm [5]. Although the RAMLA and BSREM algorithms were proved to converge, they require a strong assumption that the objective sequence is convergent.

Recently, the relaxed ordered subsets separable paraboloidal surrogates (OS-SPS) algorithm [6] has been shown to converge without the strong assumption. This algorithm is derived from the separable paraboloidal surrogates (SPS) algorithm $[7,8]$, which is closely related to the EM algorithms. Like the EM algorithms, the OS version of the SPS (OS-SPS) algorithm [9] was introduced for transmission tomography. Even though the OS-SPS algorithm converges very fast, it is not guaranteed to converge. To fix the convergence problem of the OS-SPS algorithm, the relaxed OSSPS algorithm [6] was proposed by introducing the relaxation parameter into the algorithm. This algorithm not only retains the fast convergence rate of the OS-SPS algorithm but is guaranteed to globally converge as well. Unlike the relaxed OS-SPS algorithm, the relaxed version of the OSEM algorithm is not guaranteed to converge to the optimal point. Therefore, in this paper we will focus on the relaxed OS-SPS algorithm.

Most existing OS methods have been applied to image reconstruction in tomography only, but not to image restoration. In [10], the OS-EM method has been applied to the restoration of the large binocular telescope (LBT) images. However, the structure of the LBT imaging is similar to that of the computed tomography (CT): multiple views of the same object have been observed at different angles. Since the measurement data are different in image restoration than those obtained in image reconstruction, we develop here a different strategy for choosing subsets.

\section{MEASUREMENT MODEL}

In image restoration problems, the measurements are usually degraded by blur and noise. To recover the original image, one can use the statistical characteristics of the measurement system to specify an objective function that is max- 
imized. Since image restoration is an ill-posed problem, we focus on the penalized likelihood (PL) estimation. Thus the objective function can be written in the following form:

$$
\Phi(x)=L(x)-\beta R(x)
$$

where $L$ is the $\log$-likelihood function of the measurement, $R(x)$ is the roughness penalty function, and $\beta$ is a parameter that controls the degree of smoothness in the restored image.

For confocal microscopy, the noisy measurement $Y$ can be modeled as follows:

$$
Y_{i} \sim \text { Poisson }\left\{[A x]_{i}+b_{i}\right\}, \quad i=1, \ldots, N
$$

where $A$ is the system matrix which is assumed to be known, $x$ is the unknown image that we must estimate, $b_{i}$ is the background noise and dark current, and $N$ is the number of pixels. The corresponding log-likelihood function is given by

$$
L(x)=\sum_{i=1}^{N} h_{i}\left(l_{i}\right)
$$

where $l_{i}=\sum_{j=1}^{p} a_{i j} x_{j}$ and $h_{i}(l)=y_{i} \log \left(l+b_{i}\right)-\left(l+b_{i}\right)$, ignoring irrelevant constants independent of $x$.

To reduce noise, we penalize the differences between neighboring pixels using a roughness penalty function of the form

$$
R(x)=\sum_{i=1}^{r} \psi\left([C x]_{i}\right)
$$

where $\psi$ is the potential function and $C$ is the penaity matrix. For the first-order neighborhood, the matrix $C$ consists of horizontal and vertical cliques.

With proper regularization, the objective function has a unique global maximum. Thus our goal is to estimate $x$ by finding the maximizer of the objective function: $\hat{x} \triangleq$ $\arg \max _{x \geq 0} \Phi(x)$. Since closed form solutions are unavailable for the maximizer, iterative algorithms are needed.

\section{THE ALGORITHMS}

\subsection{OS-SPS Algorithm}

In this section, we review the idea of the OS technique and the OS-SPS algorithm.

The objective function in (1) can be decomposed into subobjective function $f_{m}$ as follows:

$$
\Phi(x)=\sum_{m=1}^{M} f_{m}
$$

where $M$ is the total number of subsets and $f_{m}$ 's are obtained by replacing a sum over all pixel indices in the likelihood function of (2) with a sum over a subset of data $S_{m}$ as follows:

$$
f_{m} \triangleq \sum_{i \in S_{m}} h_{i}\left(l_{i}\right)-\frac{\beta}{M} R(x) .
$$

Suppose the "subset-balance"-like conditions [3] hold for the gradient of each sub-objective function, i.e. $\nabla f_{1}(x) \cong$ $\nabla f_{2}(x) \cong \ldots \cong \nabla f_{M}(x)$. Then the gradient of the objective function $\Phi(x)$ can be approximated as follows:

$$
\nabla \Phi(x) \cong M \nabla f_{m}(x), \quad \forall m .
$$

From (3), $M \nabla f_{m}(x)$ is replaced with $\nabla \Phi(x)$ in the algorithm to construct the OS algorithm.

The SPS algorithm is based on the paraboloidal surrogate function and the concavity technique developed by De Pierro [2]. The OS version of the SPS algorithm was introduced in [9] for transmission tomography. Using (3), the pixel update $x_{j}$ for the OS-SPS algorithm is

$x_{j}^{(n, m)}=\left[x_{j}^{(n, m-1)}+M \frac{\nabla_{j} f_{m}}{d_{j}+\beta p_{j}}\right]_{+}, m=1, \ldots, M$

where, in the PL estimation,

$\nabla_{j} f_{m}=\sum_{i \in S_{m}} a_{i j} \dot{h}_{i}\left(l_{i}^{(n, m-1)}\right)-\frac{\beta}{M} \sum_{i=1}^{r} c_{i j} \dot{\psi}\left(\left[C x^{(n, m-1)}\right]_{i}\right)$

The curvature of the likelihood $d_{j}$ and the curvature of the penalty $p_{j}$ are precomputed as follows:

$$
\begin{aligned}
& d_{j}=\sum_{i=1}^{N} a_{i j} \gamma_{i} c_{i} \\
& p_{j}=\sum_{i=1}^{r} c_{i j} \nu_{i} \omega(0)
\end{aligned}
$$

where $\gamma_{i}=\sum_{j=1}^{p} a_{i j}, c_{i}=-\ddot{h}_{i}\left(y_{i}-b_{i}\right), \nu_{i}=\sum_{j=1}^{p} c_{i j}$, and $\omega(t)=\frac{\dot{\psi}(t)}{t}$. Although the OS-SPS algorithm converges faster than SPS in early iterations, it is not guaranteed to converge.

\subsection{Relaxed OS-SPS Algorithm}

To guarantee the convergence of the OS-SPS algorithm, Ahn and Fessler [6] modified the OS-SPS algorithm to include the relaxation parameter. From (4), the pixel update of the relaxed OS-SPS algorithm becomes

$x_{j}^{(n, m)}=\left[x_{j}^{(n, m-1)}+\alpha_{n} M \frac{\nabla_{j} f_{m}}{d_{j}+\beta p_{j}}\right]_{+}, m=1, \ldots, M$

where a positive relaxation parameter $\alpha_{n}$ is chosen such that $\sum_{n} \alpha_{n}=\infty$ and $\sum_{n} \alpha_{n}^{2}<\infty$. 


\subsection{Choosing Subsets}

Since most OS algorithms have been used for image reconstruction to date, a different strategy for choosing subsets in image restoration needs to be considered because of difference in data acquisition. A good choice of subsets should satisfy the "subset-balance" condition stated in (3). In tomography, the subsets are chosen from downsampling the projection angles. One approach to obtain the subsets in restoration problem is to downsample the pixels in the image. Possible choices of four subsets for a $2 \mathrm{D}$ image are shown in Fig. 1. How the possible choices of subsets may effect the convergence rate still has to be investigated.
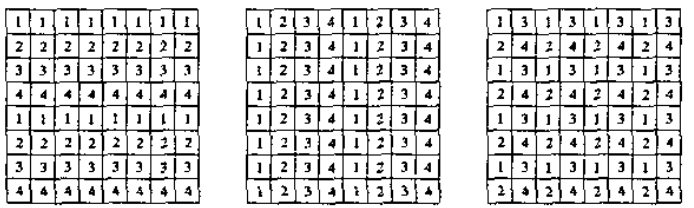

Fig. 1. Possible Choices for 4 subsets

\subsection{FFT Trick}

To increase the efficiency of computing the following expressions which produce most of the computation cost, we developed an FFT trick.

$$
\begin{aligned}
l_{i} & =\sum_{j=1}^{p} a_{i j} x_{j}, \quad \forall i \in S_{m} \\
\dot{L}_{j} & =\sum_{i \in S_{m}} a_{i j} \dot{h}_{i}\left(l_{i}\right), \quad \forall j
\end{aligned}
$$

Due to simultaneously updating all pixels, the fast Fourier transform (FFT) can be employed to reduce the computation time, especially for a large image in 3D. Since some values of $l_{i}$ in (5) are used in (6), computing all values of $l_{i}$ using ordinary FFT routines would be inefficient. Therefore, we introduced a trick for computing (5) and (6) efficiently with FFT. First, we rewrite (5) as follows:

$$
l_{i}=\sum_{m=1}^{M} \sum_{j \in S_{m}} a_{i j} x_{j}, \quad \forall i \in S_{m} .
$$

Then $\sum_{j \in S_{m}} a_{i j} x_{j}$ can be computed by downsampling the image and the point spread function (PSF) according to subset $S_{m}$ and then summing all the subsets. The size of the FFT matrix, then, is approximately reduced by the respective number of subsets. Similarly, in (6), for each $j \in S_{\mathrm{m}}$, $\dot{L}_{j}$ can be computed by using $\dot{h}_{i}$ and the downsampled PSF. A different $S_{m}$ in $\dot{L}_{j}$ requires a different downsampled PSF, but uses the same $\dot{h}_{i}$.

\section{SIMULATION RESULTS}

A $256 \times 256$ cell image (Fig. 2 a) was degraded by a $15 \times 15$ PSF, created from the XCOSM package ${ }^{1}$ (only $\mathrm{xz}$ ) [11], and Poisson noise with PSNR ${ }^{2}$ of $40 \mathrm{~dB}$, as shown in Fig. $2 \mathrm{~b}$. We assigned the relaxation parameter $\alpha_{n}=11 /(10+n)$ and for edge-preserving [12], we used the nonquadratic roughness penalty function $\psi(t)=\delta^{2}\left[\left|\frac{t}{\delta}\right|-\log \left(1+\left|\frac{t}{\delta}\right|\right)\right]$, where $\delta$ controls the degree of edge preservation. Fig. $2 c$ shows the restoration with the relaxed OS-SPS algorithm (8 subsets) performed for 50 iterations.

Table 1 compares the elapsed time per iteration of different algorithms: De Pierro's modified EM (DPEM) [2], SPS (with optimal curvature), and relaxed OS-SPS (with precomputed curvature) algorithms. Theoretically, different subsets of the relaxed OS-SPS algorithm should yield approximately the same computation time per iteration as the non OS version. Although, we were not able to achieve that due to MATLAB overhead, the computation time per iteration does not increase by the number of subsets.

\begin{tabular}{|c|c|c|c|c|c|}
\hline & DPEM & SPS & OS-SPS-2 & OS-SPS-4 & OS-SPS-8 \\
\hline ime/iter (s) & 1,09 & 1.28 & 1.62 & 2.50 & 4.27 \\
\hline
\end{tabular}

Table 1. Comparison of elapsed times per iteration for DPEM, SPS, and OS-SPS algorithms.

Fig. 3 shows the objective increase, $\Phi\left(x^{n}\right)-\Phi\left(x^{0}\right)$, at each iteration of DPEM, SPS, ordinary OS-SPS (8 subsets), and relaxed OS-SPS (8 subsets). In this figure, the ordinary OS-SPS and relaxed OS-SPS algorithms increase the objective function faster than the DPEM algorithm roughly by the number of subsets. However, the ordinary OS-SPS algorithm does not eventually converge to the same point as the relaxed OS-SPS algorithm.

\section{CONCLUSION}

We demonstrated that the relaxed OS-SPS algorithm, conventionally used for tomography, can be adapted to use in image restoration by choosing appropriate subsets. Essentially, we based this choice on the pixel location. Similarly to tomography, we are able to achieve order-of-magnitude acceleration over the nonrelaxed version algorithm. Although this preliminary study focused on 2D, our relaxed OS-SPS algorithm can be modified to include 3D confocal microscopy. The real benefit of the FFT trick is for 3D restoration. Moreover, the parallel version of FFT is also available which can further increase the convergence rate.

\footnotetext{
${ }^{1}$ pixel sizes $\Delta x=\Delta y=\Delta x=0.15 \mu \mathrm{m}, 40 \times / 1.0 \mathrm{NA}$ oil-immersion objective, and a fluorescent wavelength of $0.63 \mu \mathrm{m}$.

${ }^{2}$ The peak signal-to-noise ratio is defined as follows: PSNR $=10 \log _{10}\left(\frac{\max _{i}\left(y_{i}-b_{i}\right)^{2}}{\pi \sum_{i}\left(y_{i}-E\left[y_{i}\right)^{2}\right.}\right)$.
} 


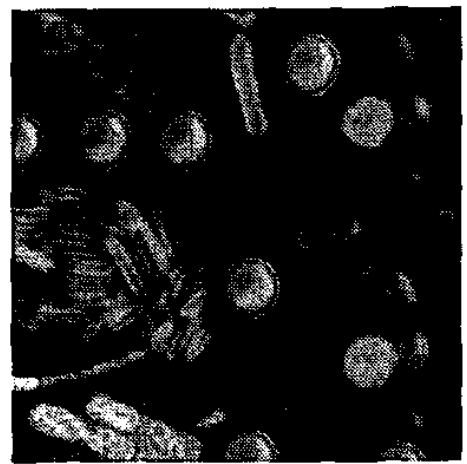

(a) Original image

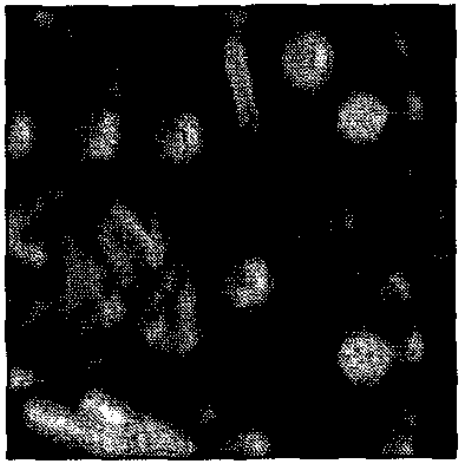

(b) Noisy image

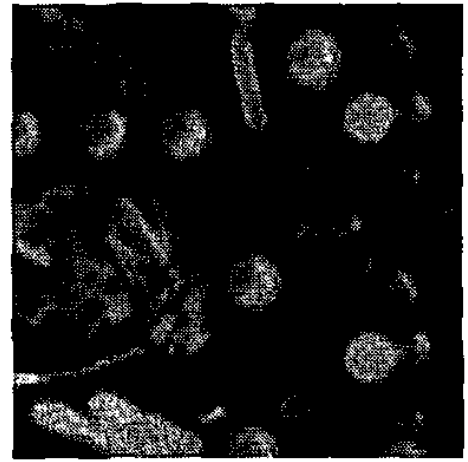

(c) Restored image

Fig. 2. Simulated images and restoration using the relaxed OS-SPS algorithm.

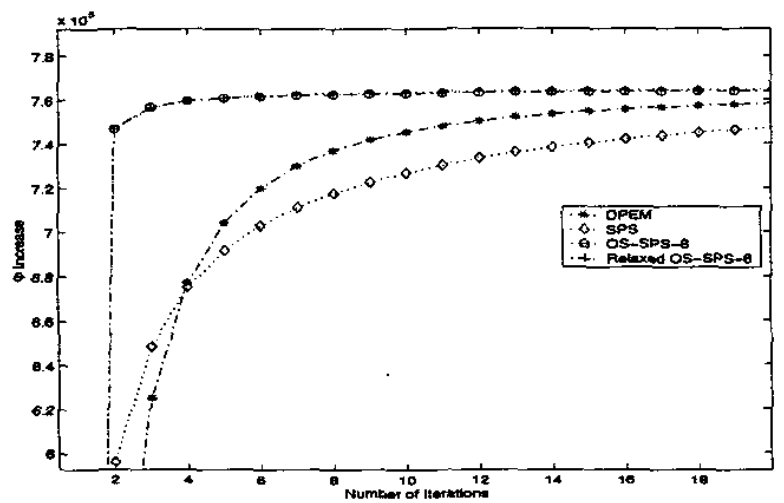

Fig. 3. Comparison of objective function increase of DPEM, SPS, OS-SPS, and relaxed OS-SPS algorithms.

\section{REFERENCES}

[1] A. P. Dempster, N. M. Laird, and D. B. Rubin, "Maximum Likelihood from Incomplete Data via the EM Algorithm," J. Roy. Stat. Soc. B, vol. 39, pp. 1-38, 1977.

[2] A. R. De Pierro, "A Modified Expectation Maximization Algorithm for Penalized Likelihood Estimation in Emission Tomography," IEEE Trans. Med. Imaging, vol. 14, no. 1, pp. 132-137, March 1995.

[3] H. M. Hudson and R. S. Larkin, "Accelerated image reconstruction using ordered subsets of projection data," IEEE Trans. Medical Imaging, vol. 13, no. 4, pp. 601-609, December 1994.

[4] J. A. Browne and A. R. De Pierro, "A row-action alternative to the EM algorithm for maximizing likelihoods in emission tomography," IEEE Trans. Med. Imaging, vol. 15, no. 5, pp. 687-699, October 1996.

[5] A. R. De Pierro and M. E. B. Yamagishi, "Fast EMlike methods for maximum 'a posteriori' estimates in emission tomography," IEEE Trans. Med. Imaging, vol. 20, no. 4, pp. 280-288, April 2001.

[6] S. Ahn and J. A. Fessler, "Globally convergent ordered subsets algorithms: Application to tomography," in Proc. IEEE Nuc. Sci. Symp. Med.Im. Conf., 2001.

[7] J. A. Fessler and H. Erdoğan, "A Paraboloidal Surrogates Algorithm for Convergent Penalized-Likelihood Emission Image Reconstruction," IEEE Nuc. Sci. Symp. and Med. Im. Conf., vol. 2, pp. 1132-1135, 1998.

[8] H. Erdoğan and J. A. Fessler, "Monotonic Algorithms for Transmission Tomography," IEEE Trans. Med. Imaging, vol. 18, no. 9, pp. 801-814, September 1999.

[9] H. Erdoğan, G. Gualtieri, and J. A. Fessler, "An Ordered Subsets Algorithm for Transmission Tomography," Phys. Med. Biol., vol. 44, no. 11, pp. 2835-51, November 1999.

[10] M. Bertero and P. Boccacci, "Application of the osem method to the restoration of LBT images," Astron. Astrophys. Suppl. Ser., vol. 144, no. 1, pp. 181-186, May 2000.

[11] The XCOSM deconvolution package [Online]. Available: http://3dmicroscopy.wustl.edu/xcosm/.

[12] K. Lange, "Convergence of EM Image Reconstruction Algorithms with Gibbs Smoothing," IEEE Trans. Med. Imaging, vol. 9, no. 4, pp. 439-446, December 1990. 